\title{
Loss of PTPN23 Promotes Proliferation and Epithelial-to-Mesenchymal Transition in Human Intestinal Cancer Cells
}

\author{
Lisa van der Lely ${ }^{a} \quad$ Janine Häfliger ${ }^{a} \quad$ Ana Montalban-Arques ${ }^{a} \quad K^{2}$ atharina Bäbler ${ }^{a}$ \\ Marlene Schwarzfischer ${ }^{a} \quad$ Max Sabev $^{\mathrm{a}} \quad$ Claudia Gottier $^{\mathrm{a}} \quad$ Silvia Lang ${ }^{\mathrm{a}}$ \\ Michael Scharl ${ }^{a, b}$ Marianne R. Spalinger ${ }^{a}$ \\ a Department of Gastroenterology and Hepatology, University Hospital Zurich, and University of Zurich, \\ Zurich, Switzerland; ${ }^{b}$ Zurich Center for Integrative Human Physiology, University of Zurich, Zurich, Switzerland
}

\section{Keywords}

Colorectal cancer · Protein tyrosine phosphatase $\cdot$ EGF receptor · Epithelial-to-mesenchymal transition · Growth factor signaling

\section{Abstract \\ Background/Objectives: Protein tyrosine phosphatase non- receptor type 23 (PTPN23) has recently been associated with several human epithelial cancers via regulation of growth factor signaling. Colorectal carcinoma (CRC) is a leading cause for cancer-related death worldwide and is associated with aberrant epidermal (EGF) and vascular endothelial growth factor signaling. Here, we investigated whether PTPN23 might play a role in CRC. Methods: Expression of PTPN23 was analyzed in CRC tissue by immunohistochemis- try. PTPN23 was silenced in HT-29 cells to address the role of PTPN23 in EGF signaling, gene expression, and cell migra- tion. Results: PTPN23 silencing in HT-29 and Caco-2 intesti- nal epithelial cancer cells significantly enhanced activation of pro-oncogenic signaling molecules and genes promoting epithelial-to-mesenchymal transition (EMT) upon EGF treat- ment, while genes encoding tight junction proteins were}

\section{KARGER}

(c) 2019 S. Karger AG, Basel

E-Mail karger@karger.com

www.karger.com/iid significantly reduced. Conclusions: Our data clearly indicate that loss of PTPN23 is associated with increased activation of pro-oncogenic signaling pathways and an enhanced ability of human intestinal cancer cells to undergo EMT. Taken together, these findings show that PTPN23 acts as a tumor suppressor gene in CRC.

c) 2019 S. Karger AG, Basel

\section{Introduction}

Colorectal carcinoma (CRC) is the third most common cancer worldwide in both men and women. Up to $70-80 \%$ of CRC develop sporadically, whereby somatic mutations mediate progression through the adenomacarcinoma sequence [1]. These mutations, such as genetic mutations in the oncogene KRAS, which is found in $40 \%$ of CRC patients, result in altered response to growth factor receptors, indicating that deregulated growth receptor signaling is crucially involved in CRC pathogenesis. Beside this, genetic syndromes such as the hereditary nonpolyposis colorectal cancer syndrome, familial adenomatous polyposis, and the Peutz-Jeghers syndrome 
make in total up to $5.1 \%$ of CRC cases [2]. Further, lifestyle factors, including a diet low in fibers and high in red meat, a high body weight, alcohol consumption, low physical activity, and advanced age are well-known risk factors for the development of CRC [1]. Most CRC cases are diagnosed in Western countries with an annual increase in incidence [3]. Whether the tumor has metastasized to lymph nodes or distant organs is a key factor in determining patient survival [4]. More than $50 \%$ are diagnosed with metastasis at or beyond stage III of the American Joint Committee on Cancer (AJCC) tumor/ node/metastasis (TNM) staging system. In case of CRC metastases, 5-year survival rate drops from 60 to $10 \%$ [5]. CRC is the fourth most common cause of cancer-related death worldwide [3], and investigation of the risk factors and molecular mechanisms involved in CRC pathophysiology is an important task to identify novel therapeutic targets.

Cells that acquire invasive and metastatic characteristics by sporadic epigenetic modifications are typical for the malignant progression of CRC. One such process, which allows cells to obtain invasive and metastatic potential, is the so-called epithelial-to-mesenchymal transition (EMT). During EMT, polarized epithelial cells lose their characteristics and undergo tight junction detachment, impairment in apical-basal polarity, and reorganization of cytoskeletal architecture, and thereby gain mesenchymal properties such as increased motility. Growth factors, inflammatory cytokines, and hypoxia regulate EMT in cancer cells, allowing the malignant cells to adapt to the tumor microenvironment and to develop an invasive and metastatic phenotype [6]. Of note, targeting epidermal growth factor (EGF) and vascular endothelial growth factor (VEGF) signaling is a well-established therapeutic approach in CRC treatment.

Protein tyrosine phosphatase (PTP) nonreceptor type 23 (PTPN23, also known as His domain-containing PTP [HD-PTP]), has recently been associated with mammary epithelial cancer development via regulation of EMT and subsequent disruption of the E-cadherin/ $\beta$-catenin association [7]. PTPN23 is a member of the PTP family, which removes phosphate groups from phosphorylated tyrosine residues on target proteins, including surface receptors and downstream signaling molecules. In that way, PTPs regulate signal transduction events involved in cell differentiation, proliferation, apoptosis, adhesion, motility, invasion, and migration $[7,8]$. Further, PTPN23 regulates EGF receptor (EGFR) cell surface expression in many cell types [9]. Of note, EGF and EGFR play an important role in wound repair and cell growth [10]. PTPN23 forms part of the endosomal sorting complexes required for transport (ESCRT) pathway, which mediates EGFR internalization and endosomal degradation. PTPN23 deletion in epithelial cervix carcinoma cells leads to a stabilization of EGFR on the cell surface likely resulting in increased downstream signaling pathway activation [9].

The gene locus encoding PTPN23 is located on the vulnerable chromosomal region $3 \mathrm{p} 21.3$, a hot spot for deletion in human epithelial cancers [11], including breast cancer and epithelial cervix carcinoma, but also testicular germ cell tumors, lung, skin, prostate, and esophagus cancers [12]. Although, the role of PTPN23 in the pathophysiology of some epithelial tumors has been described, its role in colon cancer cells has not yet been identified. Therefore, the aim of this study was to investigate the role of PTPN23 in human colonic HT-29 and Caco-2 epithelial cancer cells.

\section{Materials and Methods}

\section{Materials}

The following TaqMan genotyping assays were obtained from Thermo Fisher (Waltham, MA, USA): PTPN23 (Hs00394144_m1), CDH1 (Hs01023894_m1), ETS1 (Hs00428293_m1), ITGB6 (Hs00168458_m1), EGFR (Hs01076078_m1), occludin (Hs00170162_m1), vimentin (Hs00185584_m1), zonulin-1 (Hs01551861_m1), DKK1 (Hs00183740_m1), and SNAIL (Hs00195591_m1). Antibodies against human phosphorylated $\left(\mathrm{Tyr}^{416}\right) /$ total Src, EGFR, phosphorylated $\left(\mathrm{Tyr}^{397}\right) /$ total FAK; phosphorylated $\left(\mathrm{Ser}^{473}\right) /$ total Akt; phosphorylated $\left(\mathrm{Tyr}^{202 / 204}\right)$ extracellular signal-regulated kinase (ERK); phosphorylated $\left(\mathrm{Tyr}^{705}\right) /$ total STAT3; E-cadherin, and phosphorylated $\left(\mathrm{Ser}^{552}\right) /$ total $\beta$-catenin were obtained from Cell Signaling Technologies (Danvers, MA, USA). Anti-human PTPN23 and anti- $\beta$-actin antibodies were obtained from Sigma Aldrich (St. Louis, MO, USA), and anti-human total ERK antibody from Millipore (Burlington, MA, USA).

\section{Cell Culture}

Human colonic HT-29 and Caco-2 epithelial cancer cells were obtained from DMSZ and cultured in Dulbecco's modified Eagle's medium (DMEM; Life Technologies, Paisley, UK) supplemented with $0.1 \%$ nonessential amino acids (NEAA; Life Technologies) and $10 \%$ fetal calf serum (FCS; PAN Biotech, Brunschwig, Switzerland). Cells were grown in a $10 \% \mathrm{CO}_{2}$ atmosphere and detached using trypsin before seeding $1 \times 10^{6}$ cells/well on 12 -well tissue culture plates.

\section{siRNA Transfection}

Directly before siRNA transfection, the cell culture medium was changed to culture medium without FCS (SF-DMEM). Knockdown of PTPN23 was then achieved using 3 human PTPN23 silencer predesigned siRNA constructs from Life Technologies at a concentration of $5 \mathrm{nmol} / \mathrm{mL}$. For transfection, all 2 PTPN23-specific siRNA constructs were mixed with Lipofectamine RNAiMax (Life Technologies, Carlsbad, CA, USA) and incubated at room 
temperature for $20 \mathrm{~min}$ before adding to the cell culture media (SFDMEM). After $8 \mathrm{~h}$, medium was changed to DMEM supplemented with $0.1 \%$ MEM NEAA and $10 \%$ FCS, and cells were cultured for another $24 \mathrm{~h}$ before further treatment.

\section{Cell Activation}

For EGF (R\&D) treatment, cell culture medium was supplanted by SF-DMEM in order to minimize interference of growth factors present in the FCS with the experiments. After $8 \mathrm{~h}$, cells were stimulated with EGF $(100 \mathrm{ng} / \mathrm{mL})$ for $30 \mathrm{~min}$ for protein isolation or for $24 \mathrm{~h}$ for RNA isolation unless specified otherwise.

\section{Cell Lysates}

After stimulation, the cells were washed twice in ice-cold Dulbecco's phosphate-buffered saline (PBS, without calcium chloride and magnesium chloride; Sigma Aldrich) and lysed in the assayspecific buffer.

\section{RNA Isolation and RT-PCR}

Isolation of total RNA was performed using the RNeasy mini kit (Qiagen, Hilden, Germany) according to the manufacturer's instructions. RNA concentration was measured using a NanoDrop spectrophotometer (ND-1000; Witec AG, Littau, Switzerland). Complementary DNA (cDNA) synthesis was performed using the HighScribe reverse transcriptase kit (Applied Biosystems, Thermo Fisher). Real-time PCR was performed using FAST qPCR master mix (Thermo Fisher Scientific) on an RT-PCR QuantStudio 6 Flex system. All measurements were performed in triplicate, human GAPD (endogenous control [VIC, MGB probe], Applied Biosystems, Thermo Fisher) was used as endogenous control, and results were analyzed using the $\Delta \Delta \mathrm{C}_{\mathrm{T}}$ method. The real-time PCR contained an initial enzyme activation step $\left(5 \mathrm{~min}, 95^{\circ} \mathrm{C}\right)$ followed by 45 cycles consisting of a denaturing $\left(95^{\circ} \mathrm{C}, 15 \mathrm{~s}\right)$ and an annealing/ extending $\left(60^{\circ} \mathrm{C}, 1 \mathrm{~min}\right)$ step.

\section{Protein Isolation and Western Blots}

Proteins were isolated using mammalian protein extraction reagent (M-Per; Fisher Scientific) according to the manufacturer's instructions. Equal amounts of proteins from each lysate were loaded on polyacrylamide gels and blotted onto nitrocellulose membranes after separation by gel electrophoresis. Membranes were blocked in a 1\% BSA and 3\% milk containing blocking solution and incubated overnight in blocking solution with an appropriate concentration of primary antibody. Membranes were washed 3 times with washing buffer (Tris-buffered saline containing 1\% Tween 20) before incubation with HRP-coupled anti-rabbit secondary antibody (Lab Force, Santa Cruz, CA, USA) for $2 \mathrm{~h}$. Immunoreactive proteins were detected with a Fusion Solo $\mathrm{S}$ imager (Vilber Lourmat; Witec AG) using a Western blotting detection kit (Western Bright Sirius or ECL; Advansta, Menlo Park, CA, USA).

Immunoprecipitation and Detection of Phosphorylated EGFR

To detect EGFR phosphorylation, $150 \mu \mathrm{g}$ of protein was precleared on a shaker with Sepharose G beads (GE Healthcare, Little Chalfont, UK) for $30 \mathrm{~min}$ prior to incubation at $4{ }^{\circ} \mathrm{C}$ with $5 \mu \mathrm{g}$ anti-EGFR antibody overnight. Antibody-bound protein was then precipitated using Sepharose $\mathrm{G}$ beads according to the manufacturer's instructions and washed 3 times in ice-cold PBS; precipitates were heated in $20 \mu \mathrm{L} 1 \times$ loading buffer (Invitrogen, Carlsbad, CA, USA) for $10 \mathrm{~min}$, and supernatants were used for Western

PTPN23 Regulates EGF-Induced

Signaling in Colon Cancer Cells blots as described above. To detect phosphorylated tyrosine residues, an HRP-labeled, mouse anti-phosphotyrosine antibody (PY20; Cell Signaling Technologies) was used.

\section{Electric Cell Substrate Impedance Sensing}

Electric cell substrate impedance sensing (Applied BioPhysics, Trogy, NY, USA) is a real-time, impedance-based method used to quantify cell behavior considering cell-cell contacts; 100,000 HT29 cells/well were seeded on two $1 \mathrm{E} / 8 \mathrm{~W}$ plates (IBIDI, Planegg, Germany) and grown until confluency for 5 days. siRNA transfection was performed as described above. Two days later, medium was changed to SF-DMEM 8 h before cells were wounded by applying a current of 2,000 mA for $20 \mathrm{~s}$ at a frequency of $60,000 \mathrm{~Hz}$. Cells were washed with PBS prior to treatment with EGF (100 ng/ $\mathrm{mL}$ ). Resistance and capacitance measurements were collected in real time at $64,000 \mathrm{~Hz}$ (capacitance) and $4,000 \mathrm{~Hz}$ (resistance). Obtained values were normalized to the offset point at the time of wounding.

\section{Flow Cytometry}

For flow cytometry, HT-29 cells were seeded in 24-well plates at a density of $0.5 \times 10^{6}$ cells/well and transfected with siRNA prior to EGF treatment as described above. At indicated time points, cells were washed in ice-cold PBS, detached from the culture plate using 1\% trypsin/EDTA for $5 \mathrm{~min}$, and washed once in FCS containing culture medium to stop surface protein degradation. Cells were then washed again with PBS and incubated on ice with rabbit anti-human EGFR (Cell Signaling) and the Zombie NIR live-death discriminator for $15 \mathrm{~min}$. Cells were again washed and incubated for 10 min with AlexaFluor647-labeled anti-rabbit antibody (Cell Signaling) prior to fixation with the FoxP3 fixation/permeabilization kit from eBioscience. Cells were then incubated for another 15 min with PE-Texas Red-coupled anti Ki67 antibody (BioLegend, San Diego, CA, USA). After washing, cells were analyzed on an LSRFortessa from BD. For annexin V/propidium iodide (PI) staining to discriminate apoptotic cells, cells were detached from the culture plate as described above prior to incubation in annexin binding buffer (10 mM HEPES, $150 \mathrm{mM} \mathrm{NaCl}, 2.5 \mathrm{mM} \mathrm{CaCl}_{2}$ in PBS, pH 7.4) for 10 min. Cells were then stained with annexin V and PI (both from Thermo Fisher Scientific) for $10 \mathrm{~min}$ at room temperature prior to washing in annexin binding buffer and analysis on an BD LSRFortessa.

\section{Statistical Analysis}

Data are normalized to the respective control and presented as mean \pm SD. Statistical analyses were conducted by ANOVA followed by the Student-Newman-Keuls test or Man-Whitney U test where appropriate. $p$ values $<0.05$ were considered significant.

\section{Results}

EGF Treatment Induces PTPN23 Expression in HT-29 and Caco-2 Cells

In a first step to investigate a possible role for PTPN23 in the pathogenesis of CRC, we investigated whether EGF stimulation affects PTPN23 expression in human colonic cancer cell lines. Therefore, we treated the human colon 
HT-29

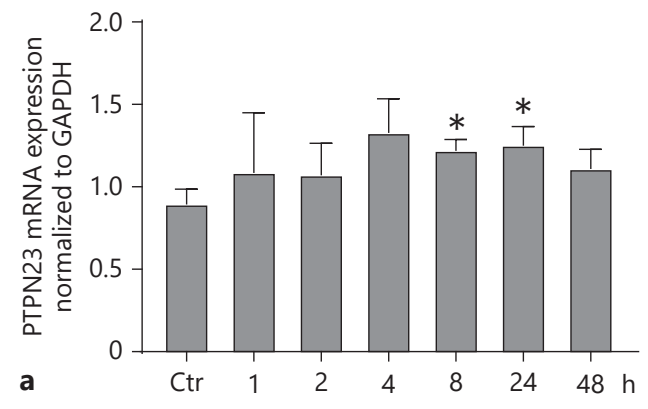

HT-29

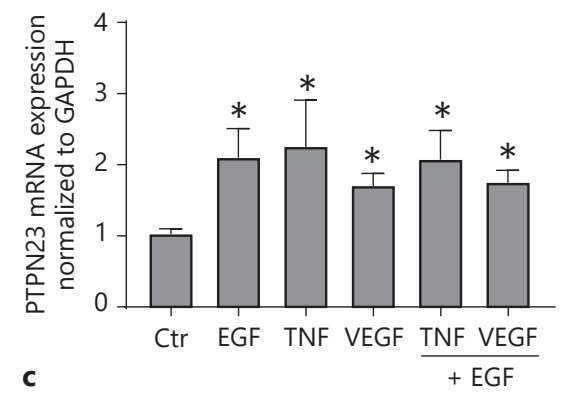

Caco-2

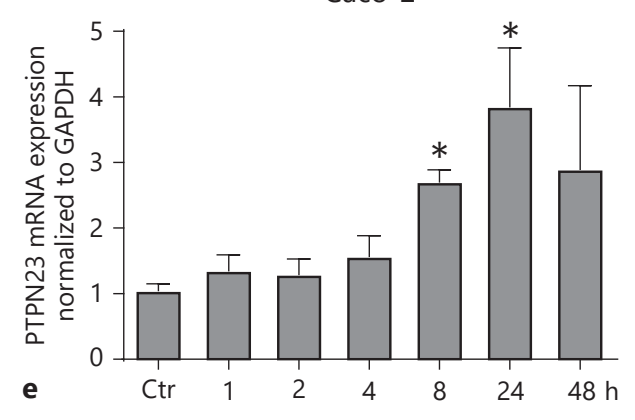

HT-29

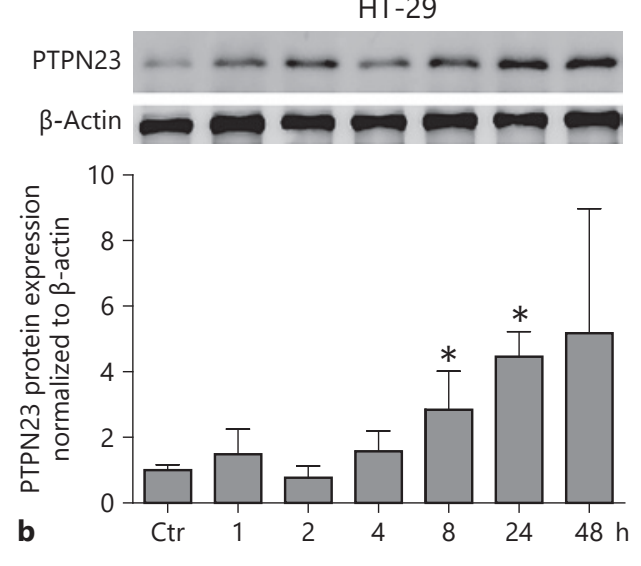

HT-29
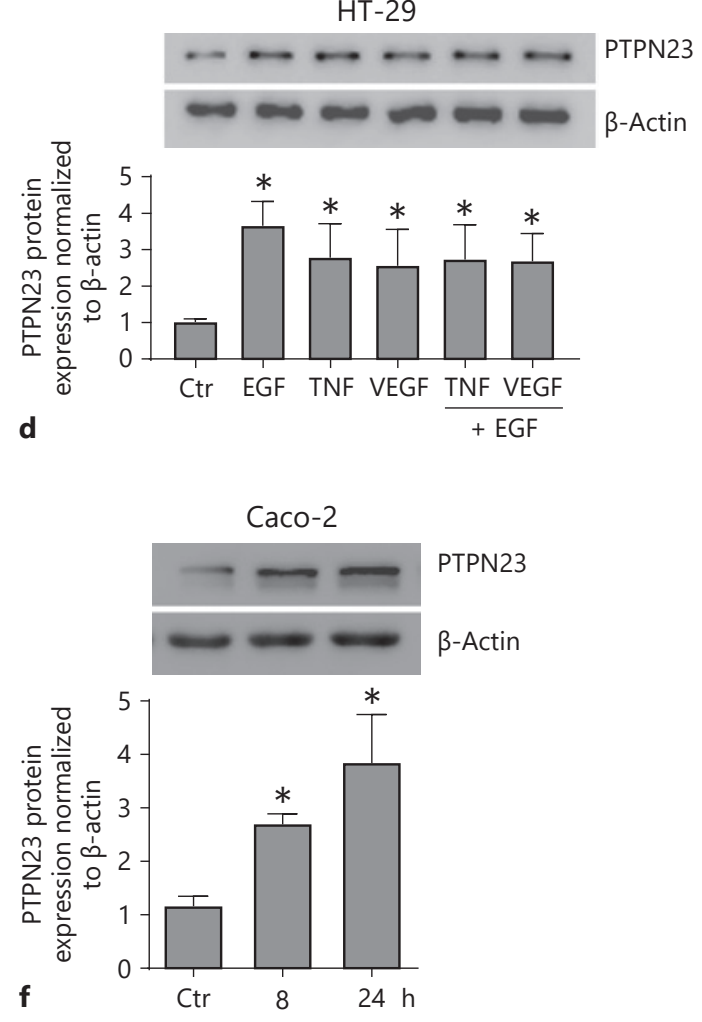

Fig. 1. Epidermal growth factor (EGF) stimulation induces PTPN23 expression. HT-29 cells were treated with $100 \mathrm{ng} / \mathrm{mL}$ EGF for the indicated times (a, b). Real-time PCR analysis shows PTPN23 mRNA expression normalized to GAPDH and untreated control (Ctr) cells (a). Representative pictures from Western blot analysis of PTPN23 protein and respective densitometric analysis (b). c, d HT-29 cells were treated with $100 \mathrm{ng} / \mathrm{mL}$ EGF, $100 \mathrm{ng} / \mathrm{mL}$ VEGF, $100 \mathrm{ng} / \mathrm{mL}$ TNF, or a combination of EGF + VEGF or EGF + TNF for $24 \mathrm{~h}$. c Real-time PCR analysis shows PTPN23 mRNA expression normalized to GAPDH and untreated control cells. d Representative pictures from Western blot analysis of PTPN23 protein and respective densitometric analysis. e, f Caco- 2 cells were treated with $100 \mathrm{ng} / \mathrm{mL}$ EGF for the indicated times. e Realtime PCR analysis shows PTPN23 mRNA expression normalized to GAPDH and untreated control cells. $\mathbf{f}$ Representative pictures from Western blot analysis of PTPN23 protein and respective densitometric analysis. Data are normalized to the average of the untreated control group and $\beta$-actin. $n=5$; mean $\pm \mathrm{SD}$; $^{*} p<0.05$, vs. untreated control cells (one-way ANOVA). 
cancer cell line HT-29 with $100 \mathrm{ng} / \mathrm{mL}$ EGF for up to $48 \mathrm{~h}$ and analyzed PTPN23 mRNA and protein expression by real-time PCR and Western blotting, respectively. As shown in Figure 1a, we found an increase in the PTPN23 mRNA level with significant differences after 8 and $24 \mathrm{~h}$ of EGF stimulation $(p<0.05)$ in HT-29 cells. Figure $1 \mathrm{~b}$ confirms a significant increase in protein expression $(p<0.05)$. These data show that EGF induces PTPN23 expression, which indicates that PTPN23 might be involved in regulating EGF-mediated signaling cascades in HT-29 cells. Of note, other factors involved in tumor growth and progression, such as tumor necrosis factor (TNF) and VEGF, did also induce PTPN23 mRNA and protein expression (Fig. 1c, d). However, co-treatment of EGF with TNF or EGF with VEGF did not further enhance PTPN23 levels (Fig. 1c, d). Of note, PTPN23 also promoted PTPN23 expression in a second colon cancer cell line, namely Caco-2 cells (Fig. 1e, f).

\section{Loss of PTPN23 Results in Decreased EGFR \\ Degradation upon EGF Treatment}

In a next step, we investigated whether EGF treatment affects cell surface EGFR localization in HT-29 and Caco2 cells and whether PTPN23 is involved in EGFR degradation. Therefore, we transfected HT-29 and Caco-2 cells with PTPN23-specific or with control siRNA; $24 \mathrm{~h}$ after siRNA transfection, cells were stimulated with $100 \mathrm{ng} / \mathrm{mL}$ EGF for the indicated times (Fig. 2a, b), and EGFR expression was analyzed by Western blotting. Figure $2 \mathrm{a}$ and $2 \mathrm{~b}$ shows a clear reduction in EGFR expression in HT-29 and Caco-2 cells after EGF stimulation at all time points. Interestingly, PTPN23 knockdown led to a significant increase in EGFR protein expression compared to control cells independent of EGF stimulation $(p<0.01)$. It has been demonstrated that loss of PTPN23 results in reduced internalization of EGFR in cervix carcinoma cells [9]. Therefore, we next analyzed surface expression of

Fig. 2. PTPN23 promotes EGFR degradation. HT-29 (a, c) and Caco-2 (b) cells were transfected with either PTPN23-specific siRNA or nontargeting control siRNA (Ctr) followed by EGF stimulation with $100 \mathrm{ng} / \mathrm{mL}$ for the indicated times. a, b Representative pictures from Western blot analysis and densitometric analysis show EGFR protein expression after 1,2, and $4 \mathrm{~h}$ of EGF stimulation compared to untreated control cells. c EGFR surface expression on HT-29 cells after $30 \mathrm{~min}$, and 1 and $2 \mathrm{~h}$ of EGF stimulation compared to unstimulated control cells was analyzed by flow cytometry. Data are representative for 3 independent experiments. $n=3$; mean $\pm \mathrm{SD} ;{ }^{*} p<0.05,{ }^{* *} p<0.01$, vs. the relative control (one-way ANOVA).

PTPN23 Regulates EGF-Induced Signaling in Colon Cancer Cells
EGFR in HT-29 cells upon silencing of PTPN23 and treatment with EGF for $30 \mathrm{~min}$, and 1, 2, and $4 \mathrm{~h}$. As expected, EGF treatment resulted in a clear reduction in EGFR surface expression already after $30 \mathrm{~min}$ (Fig. 2c).

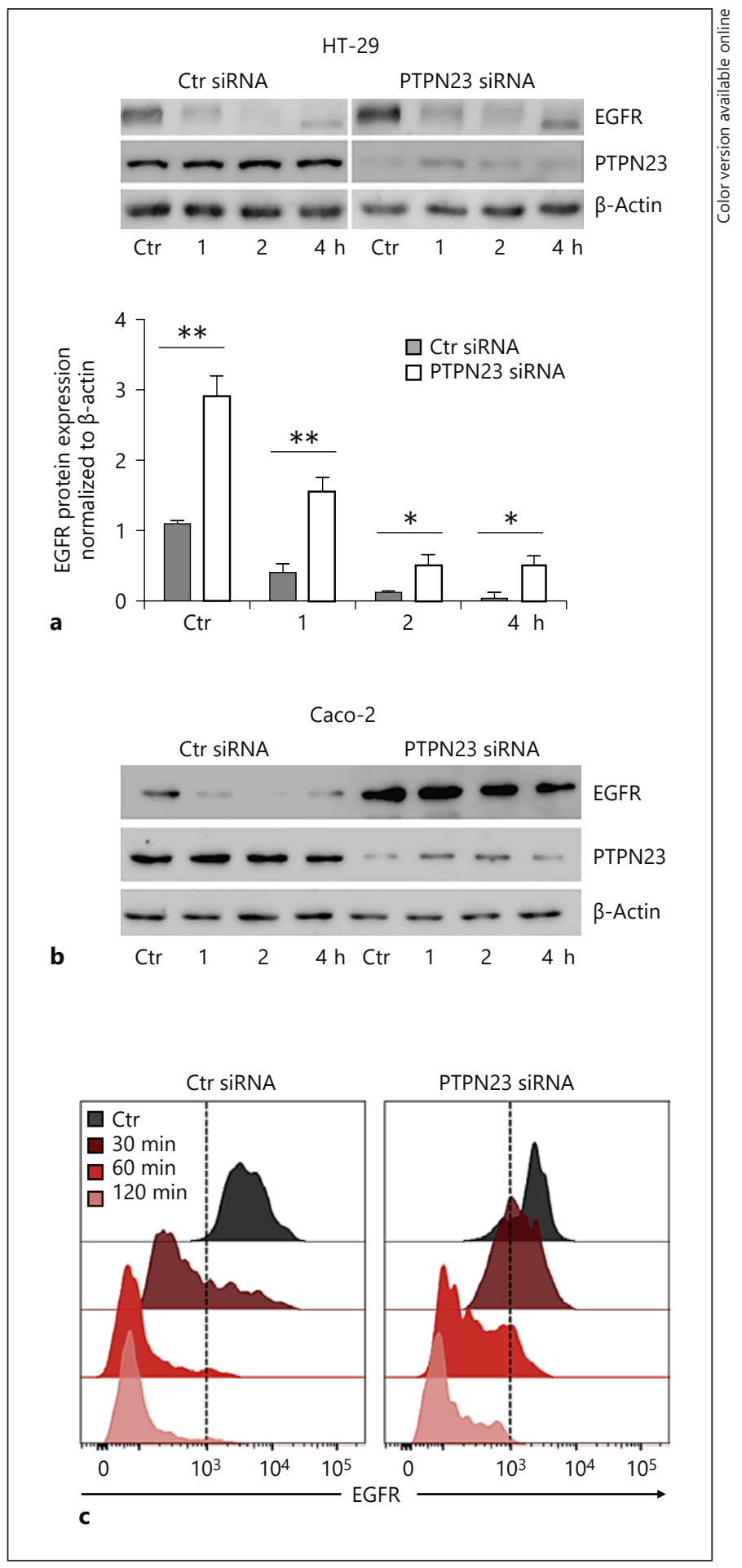

Inflamm Intest Dis 2019;4:161-173 DOI: $10.1159 / 000502861$ 


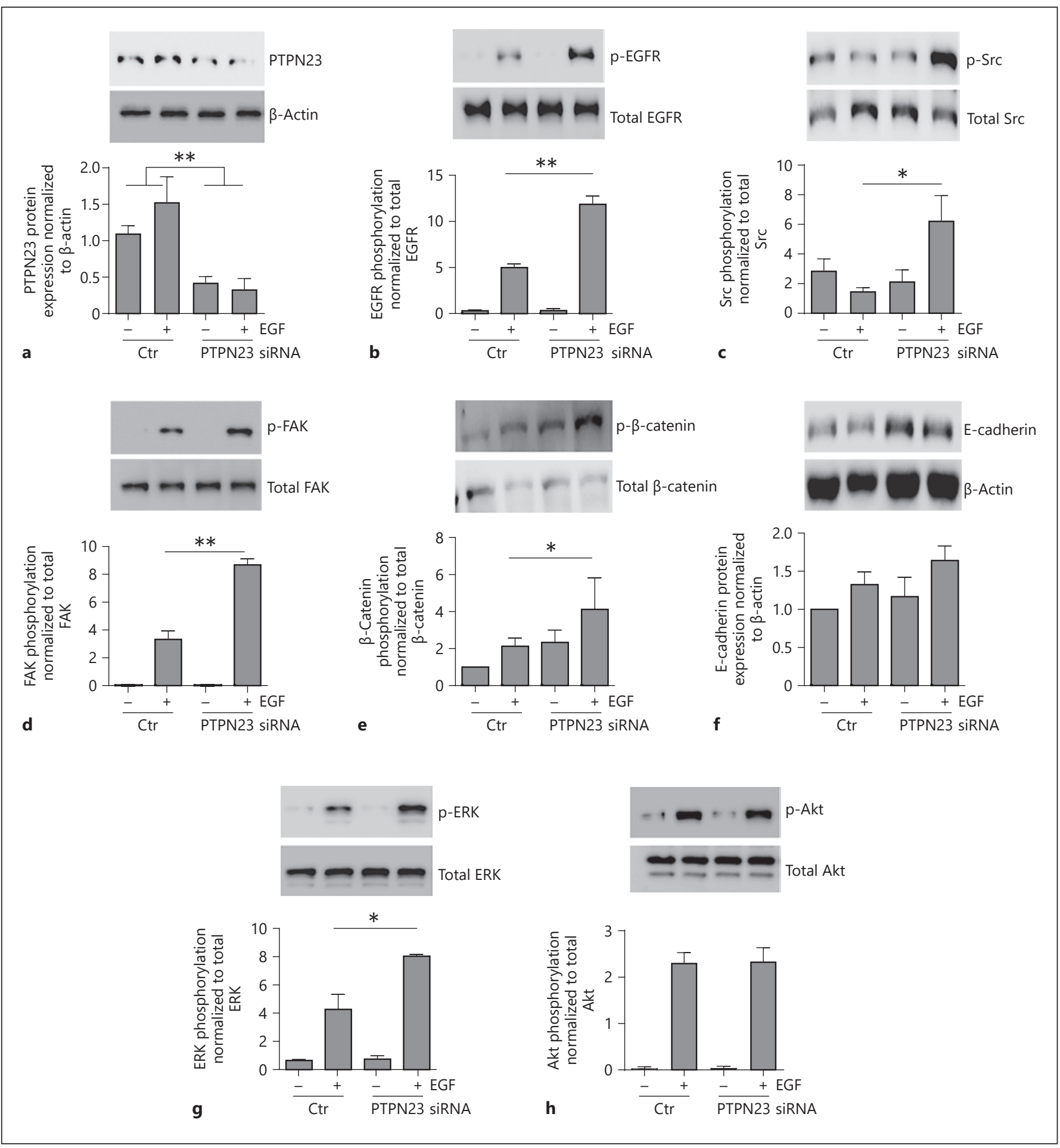

Fig. 3. PTPN23 regulates phosphorylation and thereby activation of Src, focal adhesion kinase (FAK), $\beta$-catenin, and ERK in HT-29 cells. PTPN23-specific siRNA and nontargeting control siRNAtransfected HT-29 cells were treated with EGF $(100 \mathrm{ng} / \mathrm{mL})$ for 30 $\mathrm{min}$. Shown are representative pictures from Western blot analysis and the respective densitometric analysis of PTPN23 (a); phosphorylated and total EGFR from EGFR immunoprecipitates (b); phospho- and total Src (c); phospho- and total FAK (d); phosphoand total $\beta$-catenin (e); E-cadherin (f); phospho- and total ERK protein $(\mathbf{g})$; and phospho- and total Akt (h). Data are normalized to $\beta$-actin and the untreated, control (Ctr) siRNA-transfected cells. $n=5$; mean $\pm \mathrm{SD}$; $p<0.05,{ }^{* *} p<0.01$, vs. the relative control (one-way ANOVA). 


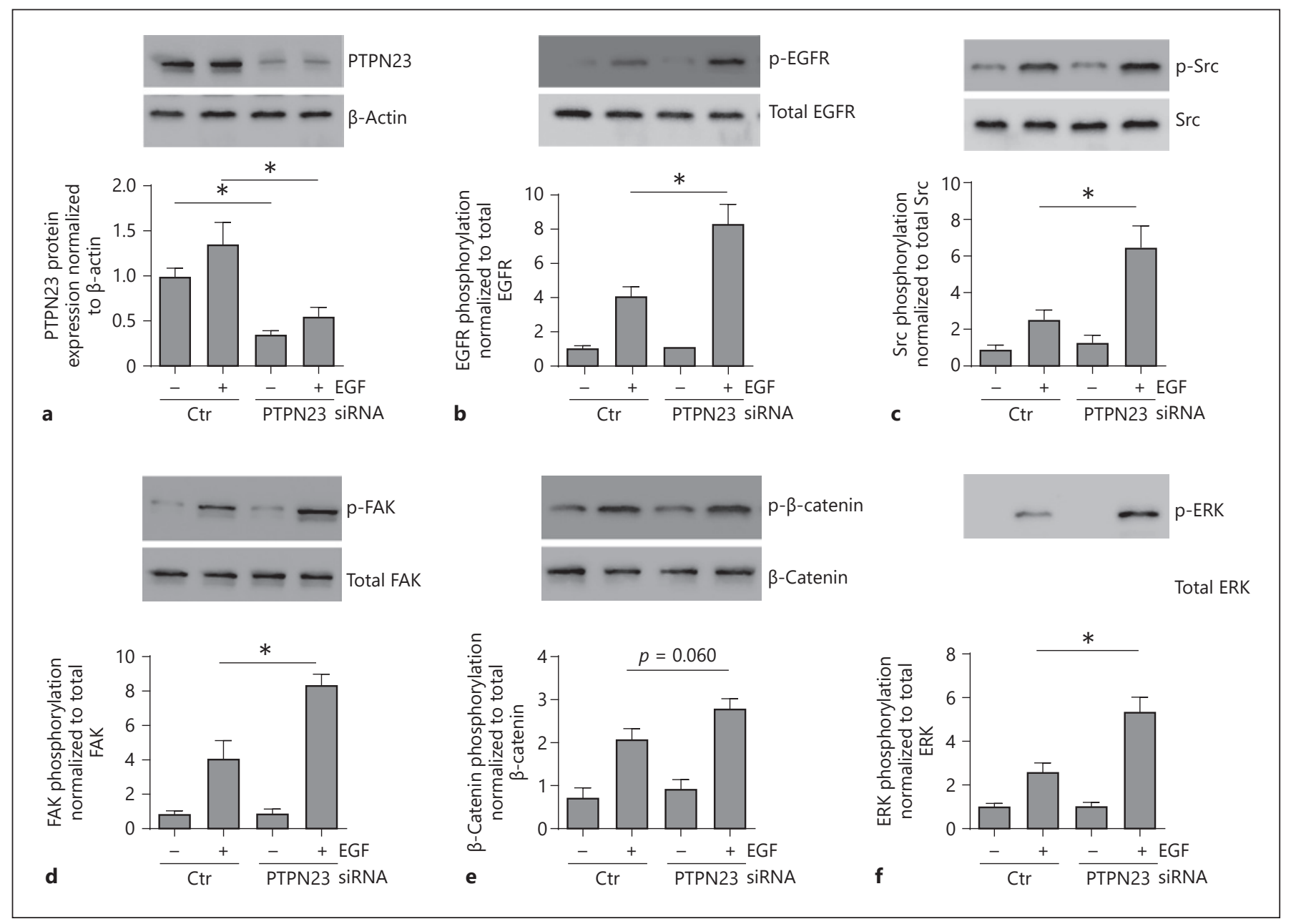

Fig. 4. PTPN23 regulates phosphorylation and thereby activation of Src, FAK, $\beta$-catenin, and ERK in Caco- 2 cells. PTPN23-specific siRNA and nontargeting control (Ctr) siRNA-transfected Caco-2 cells were treated with EGF $(100 \mathrm{ng} / \mathrm{mL})$ for $30 \mathrm{~min}$. Shown are representative pictures from Western blot analysis and the respective densitometric analysis of PTPN23 (a); phosphorylated and to-

Further, silencing of PTPN23 affected degradation of EGFR from the cell surface and led to an increase in EGFR surface stabilization after a 30-min EGF stimulation in PTPN23-knockdown cells compared to control cells. These data demonstrate that in HT-29 cells, EGFR surface expression is regulated by PTPN23. Nevertheless, we still noticed a reduction in EGFR surface expression in PTPN23-knockdown cells upon EGF presence.

\section{PTPN23 Regulates Src, FAK, $\beta$-Catenin, and ERK Activation}

Having shown that PTPN23 regulates EGFR degradation in HT-29 cells, we investigated its effect on the phos- tal EGFR from EGFR immunoprecipitates (b); phospho- and total Src (c); phospho- and total FAK (d); $\beta$-catenin (e); and representative phospho- and total ERK protein expression (f). Data are normalized to $\beta$-actin and the untreated, control siRNA-transfected cells. $n=3$; mean $\pm \mathrm{SD}$; $p<0.05$, vs. the relative control (one-way ANOVA). phorylation status of EGFR. For this aim, we transfected HT-29 cells again with PTPN23-specific siRNA, or nontargeting control siRNA. As demonstrated in Figure 3a, treatment with PTPN23-specific siRNA resulted in a clear reduction in PTPN23 protein expression. Immunoprecipitation of EGFR and subsequent analysis of tyrosine phosphorylation revealed that tyrosine phosphorylation and thereby activation of EGFR was significantly increased in PTPN23-deficient cells compared to control cells ( $p<0.01$; Fig. 3b).

We further investigated whether EGFR downstream signaling pathways were differentially activated upon PTPN23 silencing. As shown in Figure 3c and 3d, upon 


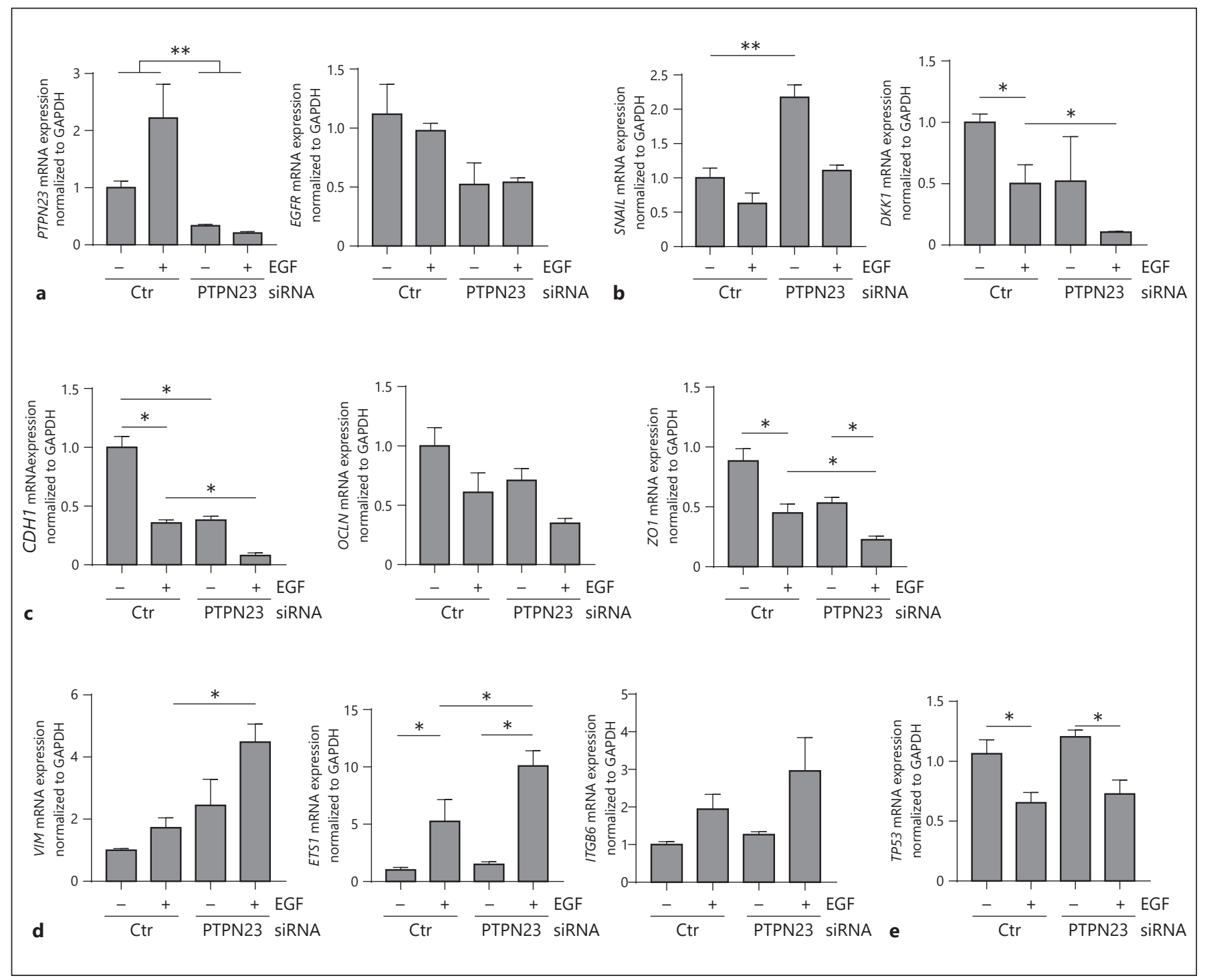

Fig. 5. PTPN23 knockdown leads to loss of tight junctions and gain of epidermal-to-mesenchymal transition (EMT) at mRNA level in HT-29 cells. HT-29 cells were transfected with either PTPN23specific siRNA or nontargeting control siRNA before treatment with EGF (100 ng/mL) for $24 \mathrm{~h}$. The graphs show mRNA levels of PTPN23 and EGFR (a); mRNA levels of SNAIL and DKK1 (b);

EGF treatment Src and focal adhesion kinase (FAK) phosphorylation are significantly higher in PTPN23-deficient cells than control cells $(100 \mathrm{ng} / \mathrm{mL}$ for $30 \mathrm{~min}, p<$ 0.05 and $p<0.01$, respectively). FAK is a cytoplasmic tyrosine kinase involved in cell migration. Further, we found significantly increased $\beta$-catenin phosphorylation in EGF-treated PTPN23-deficient cells compared to control cells ( $p<0.05$; Fig. $3 \mathrm{e}$ ), while E-cadherin protein levels were not affected in PTPN23-deficient cells (Fig. 3f).
mRNA expression of CDH1, OCLN, and ZO1 (c), and mRNA expression of VIM, ETS1, and ITGB6 (d), and TP53 (e). All data are normalized to the untreated control group and GAPDH. $n=5$; mean $\pm \mathrm{SD} ;{ }^{*} p<0.05,{ }^{* *} p<0.01$, vs. the relative control (one-way ANOVA).

ERK phosphorylation was significantly increased in PTPN23-deficient cells compared to control cells after EGF stimulation ( $p<0.05$; Fig. $3 g)$. On the other hand, surprisingly, silencing of PTPN23 did not affect EGF-induced Akt phosphorylation upon deletion of PTPN23 (Fig. 3h).

Similar results were obtained with EGF-treated Caco2 cells upon PTPN23 silencing: in these cells, we also observed enhanced levels of EGFR phosphorylation, as well 


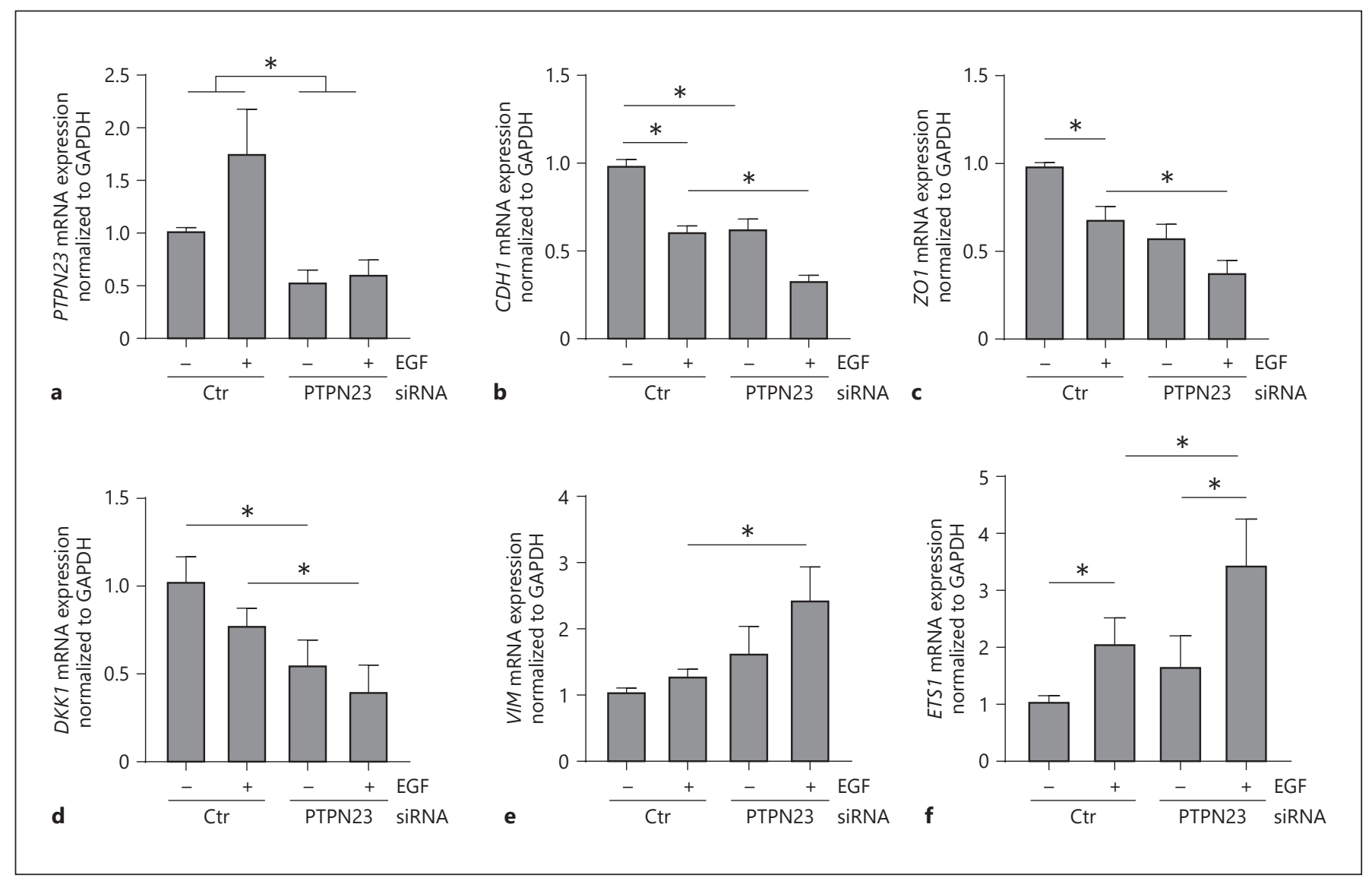

Fig. 6. PTPN23 knockdown leads to loss of tight junctions and gain of epidermal-mesenchymal transition (EMT) at mRNA level in Caco-2 cells. Caco-2 cells were transfected with either PTPN23specific siRNA or nontargeting control (Ctr) siRNA before treatment with EGF (100 ng/ml) for $24 \mathrm{~h}$. The graphs show mRNA levels of PTPN23 (a); CDH1 (b); ZO1 (c);DKK1 (d); VIM (e), and ETS1 (f). All data are normalized to the untreated control group and GAPDH. $n=3$; mean $\pm \mathrm{SD} ;{ }^{*} p<0.05$, vs. the relative control (one-way ANOVA). as downstream Src, FAK, $\beta$-catenin, and ERK phosphorylation (Fig. 4). These data indicate that PTPN23 plays a role in pro-oncogenic signaling pathways induced upon EGFR activation.

\section{PTPN23-Knockdown Leads to Loss of Tight Junctions and Gain of EMT at $m R N A$ Level}

In a next step, we addressed how silencing of PTPN23 affects mRNA expression of EGF-induced genes in HT29 cells. As expected, treatment with PTPN23 siRNA resulted in an efficient reduction in PTPN23 mRNA levels $(p<0.01$; Fig. 5a). Of note, mRNA levels of EGFR did not show a significant difference when HT-29 cells were transfected with PTPN23-specific siRNA.

Having demonstrated an increase in Src, FAK, $\beta$-catenin, and ERK activation (phosphorylation) at protein level in PTPN23-deficient cells, we now investigated the mRNA levels of their target genes. We found a significant increase in mRNA levels of the transcription factor SNAIL in nonstimulated PTPN23-deficient cells compared to control cells ( $p<0.01$; Fig. 5b). On the other hand, the $\beta$-catenin antagonist DKK1 [6] was significantly reduced in EGF-stimulated PTPN23-competent cells and even further reduced upon loss of PTPN23 compared to cells expressing normal levels of PTPN23 $(p<0.05$; Fig. 5b). We further investigated genes encoding for tight junction proteins such as $C D H 1$ (encoding E-cadherin), OCLN (occludin), and ZO1 (zonulin-1) [6]. Figure 5c shows a significant reduction in $C D H 1$ and $Z O 1$ levels in PTPN23-knockdown cells, which was further pronounced through EGF stimulation $(p<0.05)$. On the other hand, PTPN23 deletion had no significant impact on OCLN mRNA levels. At the same time, we found significantly higher mRNA levels of genes that encode mole- 

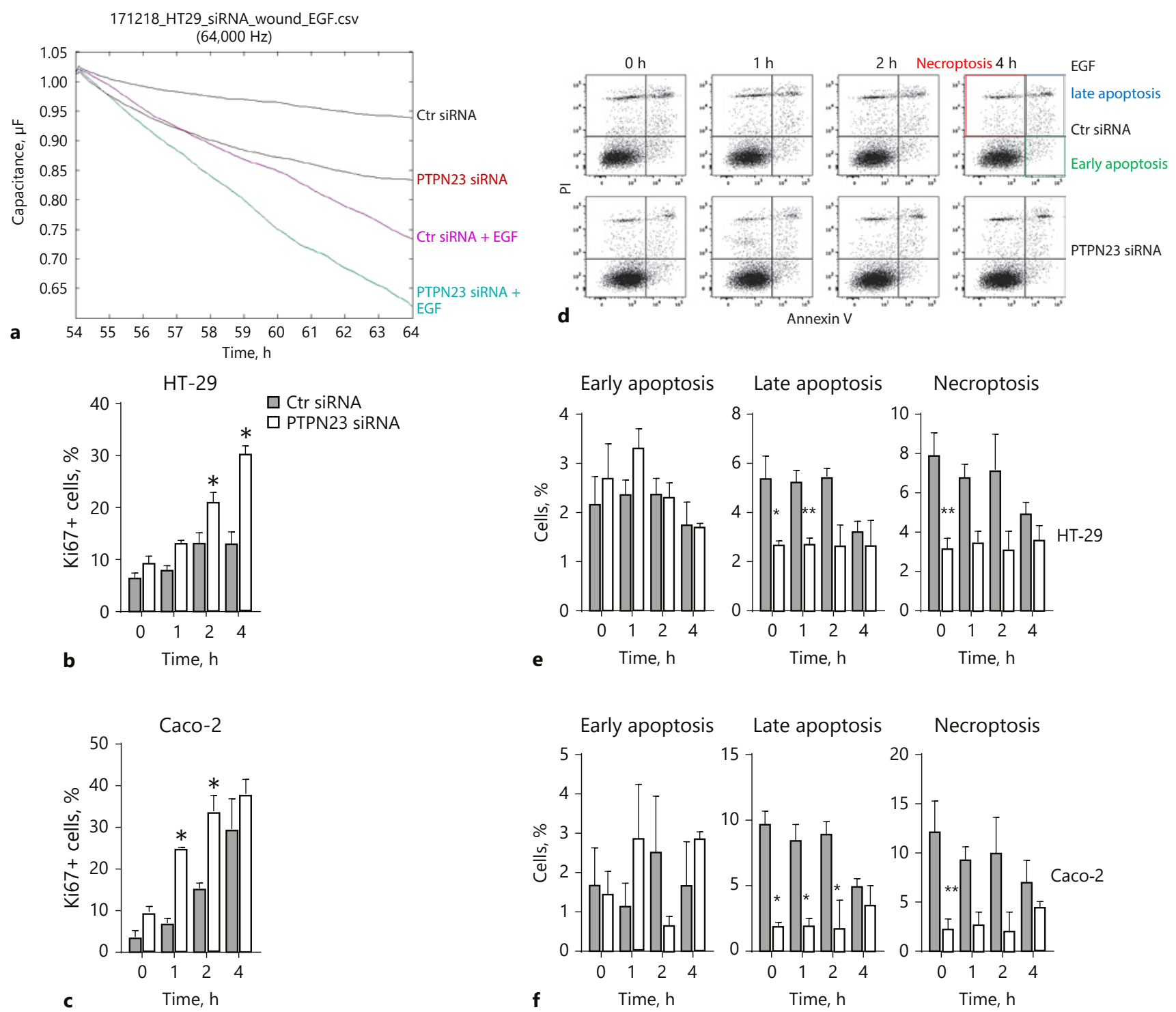

Fig. 7. Loss of PTPN23 leads to an increase in migration and proliferation and to a decrease in apoptosis. Transfection with either PTPN23-specific siRNA or nontargeting control (Ctr) siRNA was performed as described above. a Wounding with electric cell substrate impedance sensing (ECIS) was conducted prior to treatment with EGF $(100 \mathrm{ng} / \mathrm{mL})$. ECIS measured capacitance over time. Data are shown in comparison to the untreated relative control group and capacitance at the time of wounding. The lines show averages from 4 independent measurements for each condition. HT-29 (b) and Caco-2 cells (c) were stimulated with EGF (100 ng/
$\mathrm{mL}$ ) for either 1, 2, or $4 \mathrm{~h}$. The percentage of Ki67+ (proliferating) cells was analyzed by flow cytometry. Data are presented as percentage of the untreated relative control group in densitometric analysis. $\mathbf{d}-\mathbf{f}$ The rate of early and late apoptosis and necroptosis was analyzed by flow cytometry using the markers annexin $\mathrm{V}$ and propidium iodide (PI). The graph shows representative FACS dot plots (d) and statistical analysis of HT-29 (e) and Caco-2 cells (f). $n=3$; mean $\pm \mathrm{SD}$; ${ }^{*} p<0.05$, vs. relative control (one-way ANO$\mathrm{VA})$. 
cules characteristic for a mesenchymal cell phenotype, such as VIM and ETS1 ( $p<0.05$; Fig. 5d). Integrin $\beta_{6}$ (ITGB6) and tumor suppressor gene TP53 mRNA levels were not significantly altered (Fig. 5d, e). These data demonstrate a clear correlation between PTPN23 loss of function and an increase in EMT-enhancing factors at mRNA level.

Again, similar results were obtained with Caco- 2 cells, where we found reduced levels of epithelial phenotype markers, including $C D H 1, Z O 1$, and $D K K 1$, while factors associated with EMT, such as VIM and ETS1, were increased in EGF-treated Caco-2 cells (Fig. 6).

Loss of PTPN23 Results in Increased Proliferation and Migration and Decreased Apoptosis

Having demonstrated that PTPN23 deficiency impacts pro-oncogenic signaling pathways and EMT-associated gene transcription events in human HT-29 colon cancer cells, we next investigated migration and proliferation of HT-29 cells upon loss of PTPN23. To investigate how loss of PTPN23 affects cell migration and wound closure, we seeded HT-29 cells in electric cell substrate impedance sensing plates where wound closure can be assessed via capacitance measurements. Five days after seeding, when cells were $80 \%$ confluent, PTPN23 was silenced using siRNA. Two days later, a wound was induced in the epithelial layer prior to treatment with EGF (100 ng/mL), and capacitance was measured for several hours. As shown in Figure 7a, cells treated with PTPN23-specific siRNA grew much faster back to confluency than control cells, leading to a faster reduction in capacitance. Of note, EGF treatment further enhanced this effect. To confirm this finding, we next analyzed the percentage of proliferating cells by flow cytometry using the proliferation marker Ki67. Compared to control cells, loss of PTPN23 led to significantly more Ki67+ (proliferating) cells after 2 and $4 \mathrm{~h}$ of EGF stimulation ( $p<0.05$; Fig. $7 \mathrm{~b}, \mathrm{c})$. To further address whether deletion of PTPN23 affects apoptosis, we used annexin V and PI in flow cytometry. The rate of late apoptosis and necroptosis was significantly decreased in PTPN23-deficient cells compared to control cells, but this was not affected by EGF stimulation $(p<$ 0.05; Fig. 7d-f). These data indicate that PTPN23 deficiency leads to significant changes in human colonic epithelial cancer cell behavior including proliferation, migration, late apoptosis, and necroptosis.

\section{Discussion}

In the present study, we demonstrate that EGF stimulation results in increased PTPN23 expression in human colonic HT-29 and Caco-2 epithelial cancer cells and that PTPN23 in turn regulates EGF-induced signaling cascades, EMT, cell migration, and proliferation. These observations suggest for the first time that PTPN23 might be involved in the pathogenesis of colorectal carcinoma.

Besides inflammatory cytokines and hypoxia, growth factors such as EGF have been demonstrated to be elevated in the tumor microenvironment [6]. Our results indicate that increased levels of PTPN23 upon EGF treatment might protect cells from abnormally regulated EMT through dephosphorylation and thereby inactivation of EGF-induced signaling pathways. EGF treatment results in internalization and degradation of EGFR. A recent study showed a correlation between PTPN23 deficiency in epithelial cervix carcinoma cells and stabilization of EGFR on the cell surface [9]. There, Ali et al. [9] demonstrated that PTPN23 is part of the ESCRT pathway that mediates EGFR internalization and endosomal degradation. In their experiments, PTPN23 deletion resulted in diminished EGFR release from ESCRT-0 to ESCRT-III, likely resulting in increased EGFR downstream signaling pathway activation. Interestingly, we also found a correlation between PTPN23 deficiency and EGFR surface stabilization in human HT-29 cells, indicating that the role of PTPN23 as part of the ESCRT pathway is not cell type specific but rather a universal mechanism.

We have also demonstrated that PTPN23 regulates several signaling molecules downstream of EGFR. A recent publication showed that knockout of PTPN23 in mammary epithelial cells was associated with enhanced activation and autophosphorylation of FYN, a member of the Src kinase family [11]. Enhanced FYN phosphorylation upon loss of PTPN23 leads to increased phosphorylation of the cytoplasmic segment of E-cadherin and $\mathrm{Tyr}^{142}$ of $\beta$-catenin, finally resulting in the disruption of the E-cadherin/ $\beta$-catenin association [11]. As a consequence, E-cadherin is internalized in early endosomes by endocytosis, and thereby elevated FYN activation results in impaired integrity of adherens junctions. $\beta$-Catenin activation in turn increases mesenchymal protein expression and promotes EMT [7]. In our study, we demonstrate that knockdown of PTPN23 in HT-29 and Caco-2 cells leads to similar results, with significantly enhanced Src phosphorylation upon PTPN23 deletion. Src is a protein tyrosine kinase and one of the best-investigated prooncogenic molecules, which regulates major cancer-pro-

Inflamm Intest Dis 2019;4:161-173

DOI: $10.1159 / 000502861$ 
moting cellular mechanisms, including proliferation, differentiation, and survival [13]. Apart from increased Src phosphorylation, silencing of PTPN23 also promotes FAK activation. In its activated state, FAK forms a complex with Src and activates signaling pathways that promote cell migration and angiogenesis [14]. With ERK being a member of the mitogen-activated protein kinases, another signaling molecule controlling cell growth, migration and apoptosis is significantly up-regulated in PTPN23-deficient HT-29 and Caco-2 cells [15].

A further link to cell migration upon loss of PTPN23 can be drawn when considering that mRNA levels of CHD1, which encodes E-cadherin, are significantly reduced in PTPN23-deficient cells. E-cadherin is a transmembrane glycoprotein that links epithelial cells at adherens junctions and exerts a tumor-suppressive function through growth inhibition, reduction of invasiveness, apoptosis, as well as differentiation, and thereby protects from EMT. Reduced E-cadherin expression is associated with poor prognosis and survival in various human cancers, such as breast cancer and liver carcinoma [16]. Beside this, E-cadherin inhibits $\beta$-catenin and in this way dampens transcription of downstream target genes of the proliferative Wnt signaling pathway [16]. In line with a tumor suppressive role for PTPN23, we detected a significant increase in phosphorylated $\beta$-catenin protein levels in PTPN23-deficient human HT-29 and Caco-2 cells. Due to its function in enhancing Wnt signaling, $\beta$-catenin can be regarded as a proto-oncogene [17], and elevation of $\beta$-catenin has been found in sporadic CRC [18] and other tumors [17].

EMT is induced through different signaling pathways, one of them being the Wnt/ $\beta$-catenin pathway. Wnt signaling induces translocation of $\beta$-catenin to the nucleus, increases levels of SNAIL and vimentin, and suppresses the expression of E-cadherin. SNAIL is a transcription factor, which is early activated in EMT and thereby plays an important role in cancer development. Its overexpression is associated with invasiveness, metastasis, and decreased survival of CRC patients [6]. Our findings clearly point towards an increased potential for EMT in PTPN23deficient CRC cells. Upon PTPN23 knockdown, HT-29 and Caco-2 cells show a significant induction of SNAIL, while the $\mathrm{Wnt} / \beta$-catenin antagonist $D K K 1$, is significantly reduced. On the other hand, genes encoding tight junction proteins, namely CDH1 and OCLN, which are both regulated by SNAIL [6], and ZO1, which interacts with occludin to organize epithelial polarization [19], are significantly reduced. Further, genes that promote mesenchymal characteristics, such as VIM and ETS1, are significantly increased upon PTPN23 silencing - possibly indi- rectly via increased SNAIL expression/activity. Among these, increased ETS1 expression is of great interest, since its protein product, c-ets-1, contributes to cancer cell invasiveness and neo-angiogenesis, and high c-ets-1 levels are associated with poor survival in cancer patients [20].

Our data indicate that loss of PTPN23 is associated with increased activation of pro-oncogenic signaling pathways and enhanced ability of colonic epithelial cells to undergo EMT. EMT is associated with aberrant cell proliferation, reduced apoptosis, poor differentiation, increased motility, invasiveness, metastasis, and neo-angiogenesis [6]. In line with this, PTPN23-deficient HT-29 cells showed a clear increase in cell migration, a significant increase in proliferation, and significant decreases in late apoptosis and necroptosis.

Of interest, PTPN23-deficient cells showed reduced levels of apoptosis/necroptosis even without EGF stimulation. Cell preparation for flow cytometry involves mechanical dissociation of cell-cell contacts to achieve single cell suspensions, resulting in cellular stress and low levels of apoptosis since intestinal epithelial cells are sensitive to apoptosis once they lose cell-cell and extracellular matrix contacts [21], a mechanism called anoikis [22]. Reduced sensitivity to anoikis in PTPN23-knockdown cells further indicates that loss of PTPN23 promotes survival of transformed intestinal epithelial cells when they undergo EMT and dissociate from the epithelium.

Taken together, our data show that PTPN23 acts as a tumor suppressor gene in CRC. Presence of EGF results in enhanced PTPN23 expression in human colonic HT29 and Caco- 2 epithelial cancer cells, leading to a significant down-regulation of pro-oncogenic downstream signaling pathways, such as Src, FAK, $\beta$-catenin, and ERK. Upon loss of PTPN23, genes encoding tight junction proteins are significantly reduced, while EMT-enhancing genes are significantly up-regulated. Our data confirm functional changes in PTPN23-deficient human colonic HT-29 epithelial cancer cell behavior, leading to significantly increased proliferation, increased migration, and significantly decreased late apoptosis and necroptosis. This indicates that loss of PTPN23 might be involved in the transition from noninvasive tumor cells to cancer cells with metastatic potential [23]. Nevertheless, further studies are needed to address the in vivo role of PTPN23 during colon cancer development.

\section{Statement of Ethics}

This study did not involve research in human subjects or animals. 


\section{Disclosure Statement}

The authors declare no conflicts of interest.

\section{Funding Sources}

This research has been supported by grants to Michael Scharl from the Promedica Foundation, the Stiftung Experimentelle Biomedizin, and the Swiss National Science Foundation (grant No. 314730_166381).

\section{Author Contributions}

L.L., J.H., A.M.-A., K.B., M. Schwarzfischer, M. Sabev, C.G., and S.L. conducted the experiments; L.L., M. Scharl, and M.R.S. analyzed and interpreted the data; M.R.S. and M. Scharl designed and supervised the study; all authors wrote, corrected, and approved the manuscript.

\section{References}

1 Hughes LA, Simons CC, van den Brandt PA, van Engeland M, Weijenberg MP. Lifestyle, Diet, and Colorectal Cancer Risk According to (Epi)genetic Instability: Current Evidence and Future Directions of Molecular Pathological Epidemiology. Curr Colorectal Cancer Rep. 2017;13(6):455-69.

2 Witold K, Anna K, Maciej T, Jakub J. Adenomas - Genetic factors in colorectal cancer prevention. Rep Pract Oncol Radiother. 2018 Mar-Apr;23(2):75-83.

3 Mármol I, Sánchez-de-Diego C, Pradilla Dieste A, Cerrada E, Rodriguez Yoldi MJ. Colorectal Carcinoma: A General Overview and Future Perspectives in Colorectal Cancer. Int J Mol Sci. 2017 Jan; 18(1):18.

4 Peyravian N, Larki P, Gharib E, Nazemalhosseini-Mojarad E, Anaraki F, Young C, et al. The Application of Gene Expression Profiling in Predictions of Occult Lymph Node Metastasis in Colorectal Cancer Patients. Biomedicines. 2018 Mar;6(1):6.

5 McQuade RM, Stojanovska V, Bornstein JC, Nurgali K. Colorectal Cancer Chemotherapy: The Evolution of Treatment and New Approaches. Curr Med Chem. 2017;24(15): 1537-57.

$6 \mathrm{Vu} \mathrm{T}$, Datta PK. Regulation of EMT in Colorectal Cancer: A Culprit in Metastasis. Cancers (Basel). 2017 Dec;9(12):9.

7 Lin G, Aranda V, Muthuswamy SK, Tonks NK. Identification of PTPN23 as a novel regulator of cell invasion in mammary epithelial cells from a loss-of-function screen of the 'PTPome’. Genes Dev. 2011 Jul;25(13):1412-25.

8 Manteghi S, Gingras MC, Kharitidi D, Galarneau L, Marques M, Yan M, et al. Haploinsufficiency of the ESCRT Component HD-PTP
Predisposes to Cancer. Cell Rep. 2016 May; 15(9): 1893-900.

9 Ali N, Zhang L, Taylor S, Mironov A, Urbé S, Woodman P. Recruitment of UBPY and ESCRT exchange drive HD-PTP-dependent sorting of EGFR to the MVB. Curr Biol. 2013 Mar;23(6):453-61.

10 Scharl M, Rudenko I, McCole DF. Loss of protein tyrosine phosphatase N2 potentiates epidermal growth factor suppression of intestinal epithelial chloride secretion. Am J Physiol Gastrointest Liver Physiol. 2010 Oct; 299(4):G935-45.

11 Zhang S, Fan G, Hao Y, Hammell M, Wilkinson JE, Tonks NK. Suppression of protein tyrosine phosphatase $\mathrm{N} 23$ predisposes to breast tumorigenesis via activation of FYN kinase. Genes Dev. 2017 Oct;31(19):1939-57.

12 Tanaka K, Kondo K, Kitajima K, Muraoka M, Nozawa A, Hara T. Tumor-suppressive function of protein-tyrosine phosphatase non-receptor type 23 in testicular germ cell tumors is lost upon overexpression of miR142-3p microRNA. J Biol Chem. 2013 Aug;288(33): 23990-9.

13 Frankson R, Yu ZH, Bai Y, Li Q, Zhang RY, Zhang ZY. Therapeutic Targeting of Oncogenic Tyrosine Phosphatases. Cancer Res. 2017 Nov;77(21):5701-5.

14 Zhao X, Guan JL. Focal adhesion kinase and its signaling pathways in cell migration and angiogenesis. Adv Drug Deliv Rev. 2011 Jul; 63(8):610-5.

15 Yan P, Zhu H, Yin L, Wang L, Xie P, Ye J, et al. Integrin $\alpha v \beta 6$ Promotes Lung Cancer Proliferation and Metastasis through Upregulation of IL-8-Mediated MAPK/ERK Signaling. Transl Oncol. 2018 Jun;11(3):619-27.
16 Wong SH, Fang CM, Chuah LH, Leong CO, Ngai SC. E-cadherin: its dysregulation in carcinogenesis and clinical implications. Crit Rev Oncol Hematol. 2018 Jan;121:11-22.

17 Yang G, Shen T, Yi X, Zhang Z, Tang C, Wang $\mathrm{L}$, et al. Crosstalk between long non-coding RNAs and $W n t / \beta$-catenin signalling in cancer. J Cell Mol Med. 2018 Apr;22(4):2062-70.

18 Lee SK, Hwang JH, Choi KY. Interaction of the $\mathrm{Wnt} / \beta$-catenin and RAS-ERK pathways involving co-stabilization of both $\beta$-catenin and RAS plays important roles in the colorectal tumorigenesis. Adv Biol Regul. 2018 May; 68:46-54.

19 Odenwald MA, Choi W, Buckley A, Shashikanth N, Joseph NE, Wang Y, et al. ZO-1 interactions with $\mathrm{F}$-actin and occludin direct epithelial polarization and single lumen specification in 3D culture. J Cell Sci. 2017 Jan; 130(1):243-59.

20 Dittmer J. The role of the transcription factor Ets1 in carcinoma. Semin Cancer Biol. 2015 Dec;35:20-38.

21 Hausmann M, Leucht K, Ploner C, Kiessling $\mathrm{S}$, Villunger A, Becker $\mathrm{H}$, et al. BCL-2 modifying factor (BMF) is a central regulator of anoikis in human intestinal epithelial cells. J Biol Chem. 2011 Jul;286(30):26533-40.

22 Frisch SM, Francis H. Disruption of epithelial cell-matrix interactions induces apoptosis. J Cell Biol. 1994 Feb;124(4):619-26.

23 Khamas A, Ishikawa T, Shimokawa K, Mogushi K, Iida S, Ishiguro M, et al. Screening for epigenetically masked genes in colorectal cancer Using 5-Aza-2'-deoxycytidine, microarray and gene expression profile. Cancer $\mathrm{Ge}-$ nomics Proteomics. 2012 Mar-Apr;9(2):6775. 